\title{
DYNAMICAL SYSTEMS WITH CROSS-SECTIONS
}

\author{
DEAN A. NEUMANN
}

\begin{abstract}
The problem of classifying dynamical systems (flows) with global cross-sections in terms of the associated diffeomorphisms of the crosssections is considered. Suppose that, for $i=1,2, \phi_{i}$ is a $C^{r}$ flow $(r \geqslant 0)$ on the $C^{r}$ manifold $M_{i}$ that admits a global cross-section $S_{i} \subseteq M_{i}$ with associated diffeomorphism ('first return map') $d_{i}$. If rank $\left(H_{1}\left(M_{1} ; \mathbf{Z}\right)\right)=1$, then $\left(M_{1}, \phi_{1}\right)$ is $C^{s}$ equivalent $(s \leqslant r)$ to $\left(M_{2}, \phi_{2}\right)$ if and only if $d_{1}$ is $C^{s}$ conjugate to $d_{2}$. If rank $\left(H_{1}\left(M_{1} ; Z\right)\right) \neq 1$ and $\phi_{1}$ has a periodic orbit, then there are infinitely many global cross-sections $T_{i} \subseteq M_{1}$ of $\phi_{1}$, such that the associated diffeomorphisms are pairwise nonconjugate.
\end{abstract}

1. Introduction. In this paper we consider the problem of the classification of dynamical systems (flows) with global cross-sections in terms of the associated diffeomorphisms of the cross-sections.

Suppose that $S$ is a $C^{r}$ manifold (connected, but not necessarily compact and possibly with nonempty boundary; $r \geqslant 1)$ and that $d$ is a $C^{r}$ diffeomorphism of $S$. The suspension of $d$ is a $C^{r}$ flow $\phi: M \times \mathbf{R}^{1} \rightarrow M$ on an $(n+1)-$ manifold $M$ defined as follows: $M$ is the quotient space of $S \times \mathbf{R}^{1}$ obtained by identifying each point $(s, t)$ with $(d(s), t+1) ; \phi$ is the flow on $M$ induced by the constant vector field $(0,1)$ on $S \times \mathbf{R}^{1}$ (cf. [8, \$2]). We say that two diffeomorphisms, say $(S, d)$ and $\left(S^{\prime}, d^{\prime}\right)$, are flow equivalent $\left(C^{s}\right.$ flow equivalent, $1 \leqslant s \leqslant r)$ if the corresponding suspensions, $(M, \phi)$ and $\left(M^{\prime}, \phi^{\prime}\right)$, are topologically equivalent ( $C^{s}$ equivalent) (i.e., if there is a homeomorphism ( $C^{s}$ diffeomorphism) $h: M \rightarrow M^{\prime}$ that maps orbits of $\phi$ onto orbits of $\phi^{\prime}$ and preserves the natural orientation of the orbits). It is known that if $(M, \phi)$ is a $C^{r}$ flow that admits a global cross-section $S$, and $d$ is the diffeomorphism of $S$ induced by $\phi$, then $(M, \phi)$ is topologically equivalent to the suspension of $d[8$, Theorem 2.2]. Also, if $d$ and $d^{\prime}$ are topologically conjugate ( $C^{s}$ conjugate) (i.e., if $h d=d^{\prime} h$ for some homeomorphism $\left(C^{s}\right.$ diffeomorphism) $\left.h: S \rightarrow S^{\prime}\right)$, then $(S, d)$ and $\left(S^{\prime}, d^{\prime}\right)$ are $\left(C^{s}\right)$ flow equivalent. We are interested in conditions under which the converse of the latter statement is true. Our main results are stated in Theorems 1 and 2 below.

THeORem 1. Suppose that $d$ is a diffeomorphism of the $C^{r}$ manifold $S$ with suspension $(M, \phi)$ and that rank $H_{1}(M)=1$. Then $(S, d)$ is $\left(C^{s}\right)$ flow equivalent to a diffeomorphism $\left(S^{\prime}, d^{\prime}\right)$ if and only if $d^{\prime}$ is $\left(C^{s}\right)$ topologically conjugate to $d$.

Received by the editors July $25,1975$.

AMS (MOS) subject classifications (1970). Primary 58F99; Secondary 34C35, 54H 20. 
Here $H_{1}(M)$ denotes singular homology with integer coefficients. The rank of the abelian group $A$ (not necessarily finitely generated) is defined to be the maximum number of elements of $A / t A$ linearly independent over $\mathbf{Z} ; t \boldsymbol{A}$ denotes the torsion subgroup of $A$.

We obtain as a corollary of Theorem 1 the following result of G. Ikegami [5]: "If $S$ is compact and there is no homomorphism of $\pi_{1}(S)$ onto the integers, then $(S, d)$ and $\left(S^{\prime}, d^{\prime}\right)$ are flow equivalent if and only if $d$ and $d^{\prime}$ are topologically conjugate." We give examples in $\$ 3$ to show that Theorem 1 is stronger than Ikegami's Theorem.

In fact the rank condition appears to be also necessary in order that a suspension $(M, \phi)$ admit an essentially unique section. We can prove this for a large class of diffeomorphisms, but not in general:

Theorem 2. Suppose that $d$ is a diffeomorphism of the closed $C^{r}$-manifold $S$ with suspension $(M, \phi)$ and that rank $H_{1}(M) \neq 1$. If $d$ has at least one periodic point then there exist infinitely many pairwise nonconjugate diffeomorphisms $\left(S_{n}, d_{n}\right)$, each flow equivalent to $(S, d)$.

REMARK. If the Euler characteristic of $S$ is nonzero then any diffeomorphism of $S$ has a periodic point [3]. Thus for such a manifold $S$, the suspension $(M, \phi)$ of a diffeomorphism $d$ of $S$ admits a unique (up to topological conjugacy) section if and only if rank $H_{1}(M)=1$.

2. Proof of Theorem 1. Suppose that $(M, \phi)$ and $\left(M^{\prime}, \phi^{\prime}\right)$ are suspensions of $(S, d)$ and $\left(S^{\prime}, d^{\prime}\right)$ respectively, that $h: M \rightarrow M^{\prime}$ is a topological equivalence of $\phi$ with $\phi^{\prime}$, and that $\operatorname{rank} H_{1}(M)=1$. We will prove that $d$ and $d^{\prime}$ are topologically conjugate.

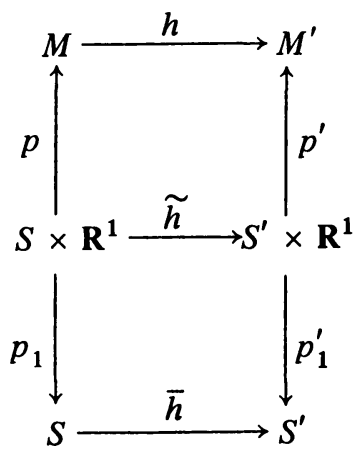

First note that the natural projection $p: S \times \mathbf{R}^{1} \rightarrow M$ is the projection of a regular covering space, with infinite cyclic group of covering transformations [7, Theorem 8.2, p. 165]. It follows that $\pi_{1}(M)$ decomposes as a semidirect product $\pi_{1}(S) \cdot \mathbf{Z}$ (the image $p_{*} \pi_{1}(S)$ is a normal subgroup of $\pi_{1}(M)$ with quotient $\mathbf{Z}$ ); similarly $\pi_{1}\left(M^{\prime}\right)=\pi_{1}\left(S^{\prime}\right) \cdot \mathbf{Z}$. Hence the commutator subgroup of $\pi_{1}(M)$ is contained in the subgroup $\pi_{1}(S)$ (i.e., $p_{*} \pi_{1}(S)$ ). Thus a generator $z$ of the infinite cyclic factor of $\pi_{1}(M)$ goes onto the generator of an infinite cyclic direct summand of $H_{1}(M)$ under the abelianizing (Hurewicz) homomor- 
phism $\alpha: \pi_{1}(M) \rightarrow H_{1}(M)$, and $\pi_{1}(S)$ is mapped by $\alpha$ onto the complementary summand. Since rank $H_{1}(M)=1$ the complementary summand is the torsion subgroup; it follows that $\pi_{1}(S)$ consists of just those elements of $\pi_{1}(M)$ that are torsion modulo the commutator subgroup. This is also true of $\pi_{1}\left(S^{\prime}\right)$ $\subseteq \pi_{1}\left(M^{\prime}\right)$ and, as this subgroup is characteristic, we see that $h_{*}: \pi_{1}(M)$ $\rightarrow \pi_{1}\left(M^{\prime}\right)$ maps $\pi_{1}(S)$ isomorphically onto $\pi_{1}\left(S^{\prime}\right)$. We can now apply the lifting criterion to obtain a homeomorphism $\tilde{h}: S \times \mathbf{R}^{1} \rightarrow S^{\prime} \times \mathbf{R}^{1}$ that makes the top rectangle in the accompanying diagram commute. Since $h$ maps orbits of $\phi$ onto orbits of $\phi^{\prime}$, it follows that $\bar{h}$ maps 'vertical' lines $\{s\} \times \mathbf{R}^{1}$ onto vertical lines. Thus $\bar{h}$ induces a homeomorphism $\bar{h}: S \rightarrow S^{\prime}$ that makes the bottom rectangle in the diagram commute.

In fact $\bar{h}$ is a conjugacy of $d$ with $d^{\prime}$. To see this let $\tau$ be the covering transformation of $S \times \mathbf{R}^{1}$ defined by $\tau(s, t)=(d(s), t+1)$, and let $\tau^{\prime}$ on $S^{\prime} \times \mathbf{R}^{1}$ be defined analogously. Then

$$
\bar{h} d(s)=p_{1}^{\prime} \tilde{h}(d(s), 1)=p_{1}^{\prime} \tilde{h} \tau(s, 0)=p_{1}^{\prime} \tau^{\prime} \tilde{h}(s, 0) ;
$$

but $\tilde{h}(s, 0) \in\left(p_{1}^{\prime}\right)^{-1}(\bar{h}(s))$, so $\tau^{\prime} \tilde{h}(s, 0) \in\left(p_{1}^{\prime}\right)^{-1}\left(d^{\prime} \bar{h}(s)\right)$; that is, $p_{1}^{\prime} \tau^{\prime} \tilde{h}(s, 0)$ $=d^{\prime} \bar{h}(s)$ as required.

If the equivalence $h: M \rightarrow M^{\prime}$ is a $C^{s}$ diffeomorphism $(1 \leqslant s \leqslant r)$, then $\tilde{h}$ is a $C^{s}$ diffeomorphism and so is $\bar{h}$; i.e., under the rank assumption, $(S, d)$ and $\left(S^{\prime}, d^{\prime}\right)$ are $C^{s}$ flow equivalent if and only if $d$ and $d^{\prime}$ are $C^{s}$ conjugate.

REMARK. Theorem 1 may be considerably extended in the case of continuous flows. Let $X$ be a connected, locally arcwise connected topological space and let $f$ be a homeomorphism of $X$. There is a continuous flow $\tilde{\phi}$ on $X \times \mathbf{R}^{1}$ defined by $\tilde{\phi}(x, s, t)=(x, s+t)$, and $\tilde{\phi}$ induces a continuous flow $\phi$ on the quotient space $Q$ obtained by identifying each point $(x, s) \in X \times \mathbf{R}^{1}$ with $(f(x), s+1)$. The argument given above carries over verbatim to prove

THEOREM $1^{\prime}$. Suppose that $f$ and $f^{\prime}$ are homeomorphisms of connected locally arcwise connected spaces $X$ and $X^{\prime}$, with corresponding suspensions $(Q$, $\phi)$ and $\left(Q^{\prime}, \phi^{\prime}\right)$. Assume that rank $H_{1}(Q)=1$. Then $\phi$ is topologically equivalent to $\phi^{\prime}$ if and only if $f$ is topologically conjugate to $f^{\prime}$.

3. Ikegami's Theorem. An immediate consequence of Theorem 1 is the following result of Ikegami [5], [6]:

Corollary. Suppose that $S$ is a closed $C^{r}$-manifold and that $\pi_{1}(S)$ admits no homomorphism onto the integers. Then $(S, d)$ and $\left(S^{\prime}, d^{\prime}\right)$ are flow equivalent if and only if $d$ and $d^{\prime}$ are topologically conjugate.

EXAMPLES. We give some examples to show that Theorem 1 is stronger than Ikegami's Theorem. In place of $S$ we take the $n$-dimensional torus $T^{n}$; let $d$ be a diffeomorphism of $T^{n}$. Then $d_{*}$ is an automorphism of the free abelian group $\pi_{1}\left(T^{n}\right) \cong \mathbf{Z}^{n}$ and hence may be represented by a matrix $A \in \mathrm{GL}_{n}(\mathbf{Z})$. (Note that any $A \in \mathrm{GL}_{n}(\mathbf{Z})$ may be obtained in this way: if $l$ is the linear homeomorphism of $\mathbf{R}^{n}$ represented by $A$, then $l$ induces a diffeomorphism $d$ of $T^{n}$, and we may choose a basis for $\pi_{1}\left(T^{n}\right)$ with respect to which $d_{*}$ is represented by $A$.)

In the semidirect product $\pi_{1}(M)=\pi_{1}\left(T^{n}\right) \cdot \mathbf{Z}$, the action on $\pi_{1}\left(T^{n}\right)$ of an (appropriately chosen) generator $z$ of the infinite cyclic factor is given by: 
$z^{-1} s z=d_{*}(s)\left(s \in \pi_{1}\left(T^{n}\right)\right)$. Hence $\pi_{1}(M)$ has presentation

$$
\left\langle e_{1}, \ldots, e_{n}, z \mid\left[e_{i}, e_{j}\right]=1 ; z^{-1} e_{i} z=d_{*}\left(e_{i}\right) ; i, j=1, \ldots, n\right\rangle,
$$

where $\left\{e_{1}, \ldots, e_{n}\right\}$ is a basis for $\pi_{1}\left(T^{n}\right)$ and $\left[e_{i}, e_{j}\right]$ denotes the commutator of $e_{i}$ and $e_{j}$. It follows that $H_{1}(M)=B \oplus \mathrm{Z}$, where $B$ has presentation

$$
\left\langle e_{1}, \ldots, e_{n} \mid\left[e_{i}, e_{j}\right]=1, e_{i}^{-1} d_{*}\left(e_{i}\right)=1, i, j=1, \ldots, n\right\rangle .
$$

We see from this that $A-I$ is a presentation matrix of $B$ (i.e., there is a free resolution $0 \rightarrow \mathbf{Z}^{n} \stackrel{i}{\rightarrow} \mathbf{Z}^{n} \rightarrow B \rightarrow 0$ of $B$, with $i$ represented by the matrix $A-I)$ and that $B$ is completely determined by the invariant factors of $A-I$. $B$ is a torsion group if and only if no invariant factor of $A-I$ is zero, i.e., if and only if 1 is not an eigenvalue of $A$. Thus we have proved:

Suppose $d$ is a diffeomorphism of $T^{n}$ such that $d_{*}$ does not have 1 as an eigenvalue. Then a diffeomorphism $d^{\prime}$ of $T^{n}$ is flow equivalent to $d$ if and only if it is topologically conjugate to $d$.

Of course, Ikegami's Theorem does not apply to any of these examples.

4. Proof of Theorem 2. Assume that $d$ is a diffeomorphism of the closed $C^{r}$ manifold $S$ with suspension $(M, \phi)$ and that $\operatorname{rank} H_{1}(M) \geqslant 2$. To find a section $S_{\eta} \subseteq M$ for $\phi$ distinct from $S$, we construct a $C^{r}$ map $P: M$ $\rightarrow S^{1} \times S^{1} ; S_{n}$ will be realized as the inverse image of a submanifold $W_{n} \subseteq S^{1} \times S^{1}$ on which $P$ is transverse regular.

We first show that there is an epimorphism $\pi: \pi_{1}(S) \rightarrow \mathbf{Z}$ satisfying $\pi d_{*}^{-1}=\pi$. Since $\pi_{1}(M) \cong \pi_{1}(S) \cdot \mathbf{Z}$ we see that $H_{1}(M) \cong B \oplus \mathbf{Z}$ and that the Hurewicz homomorphism $\alpha: \pi_{1}(S) \rightarrow H_{1}(M)$ maps $\pi_{1}(S)$ onto $B$. By computing $\pi_{1}(M)$ from Van Kampen's Theorem, we may check that a generator $z$ of the infinite cyclic factor can be chosen so that its action on $\pi_{1}(S)$ is given by: $z^{-1} \sigma z=d_{*}^{-1}(\sigma)\left(\sigma \in \pi_{1}(s)\right)$. Hence for any $\sigma \in \pi_{1}(S)$,

$$
\alpha d_{*}^{-1}(\sigma)=\alpha\left(z^{-1} \sigma z\right)=\alpha(\sigma) .
$$

Since rank $H_{1}(M) \geqslant 2$, there is an epimorphism $\beta: B \rightarrow \mathbf{Z}$; we may take $\pi=\left.\beta \circ \alpha\right|_{\pi_{1}(S)}$.

We may now construct $P$ as follows. There is a $C^{r}$ map $p: S \rightarrow S^{1}$ with $p_{*}=\pi$. Since $p_{*}=p_{*} d_{*}^{-1}$ we see that there is a homotopy $p_{t}: S \rightarrow S^{1}(t \in I$ $=[0,1])$ of $p$ to $p d^{-1}$. By $\left[4\right.$, Lemma 2] we may assume that $\left\{p_{t}\right\}$ defines a $C^{r}$ map of $S \times I$ onto $S^{1}$ and that $p_{t}=p$ for $t \in[0,1 / 3]$ and $p_{t}=p d^{-1}$ for $t \in[2 / 3,1]$. Then the map $(s, t) \rightarrow\left(p_{t}(s), t\right)$ of $S \times I$ onto $S^{1} \times I$ is compatible with the identifications $(s, 0) \leftrightarrow(d(s), 1)$ on $S \times I$ and $(s, 0) \leftrightarrow(s, 1)$ on $S^{1} \times I$, and hence induces a $C^{r} \operatorname{map} P: M \rightarrow S^{1} \times S^{1}$.

We now want to determine a condition on a 1-dimensional submanifold $W \subseteq S^{1} \times S^{1}=T^{2}$ in order that $P$ be transverse regular on $W$. On both $M$ and $T$ we consider only local coordinates that respect the "product" structure; viz., on $M$ we choose coordinates of the form $(x, t)$, where $x=\left(x_{1}, \ldots, x_{n}\right)$ are local coordinates on $S$ and $t \in J$ (open) $\subseteq I$ (corresponding to a product chart on $S \times \mathbf{R}^{1}$ ), and on $T^{2}$ coordinates of the form $(s, t), t \in J$ (open) $\subseteq I$. With respect to the corresponding bases $\left\{\partial / \partial x_{1}, \ldots, \partial / \partial x_{n}, \partial / \partial t\right\}$ of the 
tangent space $T_{(x, t)} M$ and $\{\partial / \partial s, \partial / \partial t\}$ of $T_{P(x, t)} T^{2}$, the derivative $d P$ of $P$ is represented locally by the matrix

$$
\left[\begin{array}{cccc}
\frac{\partial p_{t}}{\partial x_{1}} & \cdots & \frac{\partial p_{t}}{\partial x_{n}} & \frac{\partial p_{t}}{\partial t} \\
0 & \cdots & 0 & 1
\end{array}\right],
$$

so that the tangent vector $(0, \ldots, 0,1)$ (i.e., $\partial / \partial t)$ in $T_{(x, t)} M$ is taken onto $\left(\partial p_{t} / \partial t, 1\right)$ in $T_{P(x, t)} T^{2}$. If we fix finite covers of $M$ and $T^{2}$ consisting of charts of the above form, then

$$
m=\sup \left|\partial p_{t} / \partial t\right|<\infty
$$

(the supremum taken over all local representations of $P$ with respect to these fixed charts). The condition that $P$ be transverse regular on $W \subseteq T^{2}$ at $w \in W$ is that for any $(x, t) \in P^{-1}(w)$ we have $\left(\partial p_{t} / \partial t, 1\right) \notin T_{w} W$. Thus $P$ will be transverse regular on $W$ if, for all $w \in W$ and $(a, b) \in T_{w} W$, we have $|b / a|>m$. It is clear that, for all sufficiently large integers $k$, there is a $C^{r}$ simple closed curve $W_{k} \subseteq T^{2}$ that satisfies this condition and winds $k$-times around the $S^{1} \times\{0\}$ factor of $T^{2}$ and once around the $\left\{s_{0}\right\} \times S^{1}$ factor.

Now fix such a submanifold $W_{k} \subseteq T^{2}$ and let $S_{k} \subseteq M$ denote $P^{-1}\left(W_{k}\right)$. Since $P$ is transverse regular on $W_{k}, S_{k}$ is a codimension one $C^{r}$-submanifold of $M$ that is transverse to the flow $\phi$ [1, Theorem 17.1]. It is also true that $S_{k}$ is connected. We may see this as follows: $T^{2}$ fibers over $S^{1}$ with fibers simple closed curves "parallel" to $W_{k}$; let $q: T^{2} \rightarrow S^{1}$ denote the projection of such a fibering and let $Q=q \circ P$. We may assume that $P$ is transverse regular on each fiber of $q$, and hence that each point of $S^{1}$ is a regular value of $Q$. It follows that $Q$ fibers $M$ over $S^{1}$ with each fiber diffeomorphic to $S_{k}$ (cf. [2, $\S 1.1])$. Since $q_{*}: \pi_{1}\left(T^{2}\right) \rightarrow \pi_{1}\left(S^{1}\right)$ and $P_{*}: \pi_{1}(M) \rightarrow \pi_{1}\left(T^{2}\right)$ are surjective, so is $Q_{*}$. Because $M$ is connected and $Q_{*}$ is surjective, we see that $S_{k}$ is connected.

To prove that $S_{k}$ is a global section for $\phi$ we must show that for any $s \in S_{k}$ there is a time $\tau>0$ with $\phi(s, \tau) \in S_{k}[8, \S 2]$. We will need slightly more than this to see that the diffeomorphism $d_{k}$, induced on $S_{k}$ by $\phi$, is not conjugate to $d$; viz., that, for all sufficiently large $k$, each orbit of $\phi$ crosses $S_{k}$ at least some fixed number $j \geqslant 2$-times between successive crossings of $S$. But if $k$ is large enough then $W_{k}$ meets each "vertical" segment $s^{\prime} \times[0,1 / 3]$ at least $j$-times in $S^{1} \times[0,1 / 3]$. It follows that each orbit segment $s \cdot[0,1 / 3]=\{\phi(s, t) \mid t \in[0,1 / 3]\}$, with $s \in S$, meets $S_{k}$ at least $j$-times, as asserted.

We now make use of our assumption that $d$ has at least one periodic orbit. Let $m\left(m_{k}\right)$ be the minimal period of periodic orbits of $d\left(d_{k}\right)$. We have proved that $m_{k} \geqslant j \cdot m$, and hence that $(S, d)$ and $\left(S_{k}, d_{k}\right)$ are not topologically conjugate.

\section{BIBLIOGRAPHY}

1. R. Abraham and J. Robbin, Transversal mappings and flows, Benjamin, New York and Amsterdam, 1967. MR 39 \#2181.

2. W. Browder and J. Levine, Fibering manifolds over a circle, Comment.Math. Helv. 40 (1966), 153-160. MR 33 \#3309. 
3. F. B. Fuller, The existence of periodic points, Ann. of Math. (2) 57 (1953), 229-230. MR 14, 669.

4. S. T. Hu, On singular homology in differentiable spaces, Ann. of Math. (2) 50 (1949), 266-269. MR 10, 728.

5. G. Ikegami, On classification of dynamical systems with cross-sections, Osaka J. Math. 6 (1969), 419-433. MR 42 \#1131.

6. Flow equivalence of diffeomorphisms. I, II, Osaka J. Math. 8 (1971), 49-69, 71-76. MR 44 \# 4780.

7. W. S. Massey, Algebraic topology: An introduction, Harcourt, Brace \& World, New York, 1967. MR 35 \#2271.

8. S. Smale, Stable manifolds for differential equations and diffeomorphisms, Ann. Scuola Norm. Sup. Pisa (3) 17 (1963), 97-116. MR 29 \#2818b.

Department of Mathematics, Bowling Green State University, Bowling Green, Ohio 43403 